\title{
MIGRACIÓN FRONTERIZA BOLIVIANXS EN TALLERES TEXTILES DE BUENOS AIRES Y SAO PAULO
}

\author{
TRANSBOARDER MIGRATION \\ BOLIVIXNS IN TEXTILES FACTORIES OF BUENOS AIRES AND SÃO \\ PAULO
}

Alfonso Hinojosa Gordonava ${ }^{1}$

Postgrado en Ciencias del Desarrollo, La Paz, Bolívia

\begin{abstract}
Resumen: La histórica emigración de mano de obra boliviana hacia Argentina y Brasil, a la para que se halla afincada en matrices culturales de vieja data, hoy en día presenta características novedosas y articulaciones transnacionales que determinan el copamiento de algunos nichos laborales (los talleres textiles) y el manejo de espacios de comercialización y reproducción sociocultural de significativa importancia en las ciudades de Buenos Aires y Sao Paulo. Todo ello en medio de dinámicas de movilidad social transnacional que van aparejadas con una lenta construcción de sujetos políticos transnacionales.
\end{abstract}

Palabras-llave: Emigración; Transnacionalidad; integración social.

\begin{abstract}
The historical migration of bolivians workers to Argentina and Brasil, although is based on old cultural patterns, nowadays presents new features and transnational articulations with some work places (the textiles factories) and with spaces of cultural negotiation and reproduction of importance in the cities of Buenos Aires and São Paulo. All of this in the middle of transnational social movements connected to a slow construction of transnational political subjects.
\end{abstract}

Keywords: Emmigration; Transnationality; Social integration.

1 Pesquisador del Postgrado en Ciencias del Desarrollo (CIDES), Universidad Mayor de San Andres. E-mail: <alfhg67@gmail.com>. .Recibido en: 16.3.2016; acepto en: 07.06.2016. 


\section{INTRODUCCIÓN}

La migración fronteriza boliviana hacia Argentina y Brasil es histórica y se halla anclada en matrices culturales y dinámicas poblacionales fuertemente arraigadas en Los Andes con elementos muy marcados de ruralidad/comunidad, etnicidad y migración interna hacia las ciudades $^{2}$. En este sentido, el hecho migratorio en Bolivia no expresa solamente la puesta en práctica de estrategias de sobrevivencia modernas, sino que se trata de un habitus de movilidad espacial que les permite desplazamientos muy activos a estas sociedades dinámicas.

En un primer momento de la historia migratoria hacia Argentina, ésta se caracterizó por una mayor composición de origen indígena (en sus inicios quechua y posteriormente aymara), y por el liderazgo de los varones, especialmente en la fase rural-rural fronteriza y luego ruralurbana; estas dinámicas poblacionales alcanzaron progresivamente a la capital argentina en la segunda mitad del siglo XX, fase en que las mujeres se sumaron a los varones insertándose poco a poco en el mercado laboral de destino, pasando a un establecimiento más definitivo en el Área Metropolitana de Buenos Aires e insertos sobre todo en el trabajo textil. Por su parte, la migración hacia el Brasil, si bien es más reciente - con intensidad a partir de finales de la década de los años ochenta y con una mayor presencia de indígenas de tierras altas- se caracteriza también por su magnitud y vigor en las últimas décadas, principalmente atrayendo sectores aymaras del altiplano boliviano hacia nichos laborales de la manufactura (talleres textiles) asentados en Sao Paulo donde esta presencia es muy significativa.

En ambos países los residentes bolivianos han activado y/o formado desde su llegada redes, asociaciones y/u organizaciones de fuerte arraigo que involucran en un primer momento dimensiones laborales y económicas, aunque su sustento está dado básicamente desde lo cultural y lo comunitario, tanto en los lugares de origen como de destino y que con el transcurso del tiempo ha ido abarcando ámbitos políticos y de conquista ciudadana expresados no sólo en momentos electorales, sino también en esferas productivas y comerciales.

Si bien la mayoría de estas asociaciones de residentes en el exterior parte de la dimensión cultural que articula lo festivo (baile, música, comida, fútbol), retroalimentando y reconfigurando a la vez la identidad nacional de lo boliviano al igual que identidades más locales, es importante destacar que en las últimas décadas se viven dinámicas de acumulación y movilidad social entre estos sectores migrantes bolivianos en los nichos laborales de la manufactura textilera en las ciudades de Buenos Aires y Sao Paulo. Esa movilidad social se evidencia tanto en rubros laborales ligados al trabajo productivo rural (horticultura en la Argentina) como en el trabajo manufacturero urbano de los talleres textiles (tanto en Buenos Aires como en Sao Paulo), pero en ambos casos siempre articulados con la esfera de lo comercial, ya sea en el acopio, distribución

2 Esta perspectiva cultural en los Andes nos muestra que, desde tiempos pre-hispánicos, las culturas que habitaban y habitan el altiplano y sobre todo los valles centrales bolivianos, han mantenido una cosmovisión espacio céntrica que se manifestaba en su permanente movilidad y utilización de diferentes espacios geográficos y pisos ecológicos. La teoría de la complementariedad vertical ecosimbiótica (Condarco, 1971; Murra, 1975) hace referencia a las ancestrales y emblemáticas prácticas de movilidad poblacional gestadas en las sociedades andinas desde tiempos milenarios y que posibilitaron el surgimiento de enclaves prósperos y niveles de desarrollo avanzados. 
y/o venta al detalle de hortalizas o bien manteniendo un puesto de venta en de prendas de vestir eventos como la Feria de la Salada ${ }^{3}$ en Buenos Aires o en iniciativas similares en Sao Paulo.

En todo caso podemos afirmar que son las asociaciones y/u organizaciones de residentes bolivianos en el exterior las instancias que en buena parte constituyen el eje articulador de la bolivianeidad, no sólo en las actividades socio-culturales y económicas productivas de dichos migrantes, sino también se convierten en los espacios estratégicos a partir de los cuales podemos reconocer algunas dinámicas de movilidad social en los lugares de destino y -por ende- en los lugares de origen gozar de mayor prestigio.

\section{MIGRACIÓN TRANSFRONTERIZA BOLIVIANA A LA ARGENTINA Y EL BRASIL}

En Bolivia los procesos emigratorios fronterizos han sido una constante durante muchos años. Algunos de ellos incluso pueden ser considerados históricos, como es el caso de la migración laboral boliviana a la Argentina, cuyos orígenes y magnitudes pueden ser rastreados desde hace más de un siglo atrás, aunque los momentos de masificación corresponden a las dos últimas décadas del siglo pasado. Si bien son muchos y muy variados los destinos emigratorios de los bolivianos en el exterior, durante las últimas décadas estos procesos focalizaron como lugares de destino masivo básicamente a cuatro países: Argentina, Estados Unidos, Brasil y recientemente España.

Uno de los problemas al estudiar el hecho migratorio en Bolivia tiene que ver con la obtención de datos oficiales sobre la cantidad de residentes en el exterior. En todo caso, la cuantificación de la población boliviana en situación migratoria es una incógnita que suele ser utilizada desde diversos espacios (políticos, económicos, sociales, etc.) ya sea para magnificar, ya sea para invisibilizar o bien para dramatizar unas realidades que hasta hoy se nos presentan con demasiados vacíos.

En el año 2012 se realizó en Bolivia el Censo Nacional de Población y Vivienda en el cual por primera vez se incorporó una pregunta sobre el tema emigración internacional. La pregunta indagaba si en los hogares, durante los últimos 10 años, algún miembro había emigrado al exterior, si la respuesta era positiva se abrían otras preguntas respecto al país de residencia de esa persona, su edad y sexo. Los resultados arrojados por el censo dan un total de 487.995 bolivianos que entre el 2002 al 2012 habría emigrado del país, primando ligeramente las mujeres con un $51.03 \%$, frente a los varones con un $48.97 \%$. El destino principal de los emigrantes bolivianos en este periodo fue la república de la Argentina con un 38,22\%, seguido de España con un 23,88\%, el Brasil con 13,18\%, Chile con 5,95\% y Estados Unidos con 4,20\%. El restante 20 por ciento se dispersa en un abanico muy amplio de países entre los que sobresalen Italia, Cuba, Perú, Reino Unido, Suiza y Japón (INE, 2014).

3 La Feria Comercial de "La Salada" es uno de los mayores espacios comerciales a cielo abierto de Sudamérica. El Conglomerado de Ferias de La Salada se encuentra ubicado en un predio de más de 20 hectáreas en la localidad de Ingeniero Budge, una de las zonas más pobres del partido de Lomas de Zamora (provincia de Buenos Aires) 
Si bien estos datos reflejan en términos generales las percepciones que se tenían sobre los destinos emigratorios, en términos de cuantificación los datos son bastante inferiores a las cifras que se manejaban con anterioridad. Es claro que el umbral temporal que la pregunta del censo establecía (últimos 10 años) puede ser una causal de ello, más aun tomando en cuenta el carácter estructural de las dinámicas migratorias en Bolivia y su sostenido crecimiento desde mediados de los años ochenta. De hecho, si recurrimos a los datos que desde la Cancillería boliviana (DGRC, 2010) se manejan para los cuatro destinos internacionales más importantes tendríamos que en Argentina habría alrededor de un millón 200 mil bolivianos; en España, 240 mil personas; y en Estados Unidos y Brasil, más de 200 mil bolivianos en cada uno. En todo caso, diversas fuentes (OIM, PMH) señalan que cerca de un 20 por ciento de la población boliviana se halla más allá de sus fronteras.

Un aspecto fundamental al momento de considerar los procesos de movilidad poblacional en Bolivia tiene que ver con la necesidad de vincular el estudio de la migración interna (ya sea campo ciudad o urbana-urbana) con la migración internacional, en tanto proceso continuo e histórico, donde lo rural se halla en lo urbano y lo urbano es rápidamente incorporado a circuitos migratorios transnacionales contemporáneos. En lo que hace a la migración interna en Bolivia, cobra importancia analizar los procesos de rápida urbanización que se desarrollan desde hace más de dos décadas, con la consolidación de tres áreas metropolitanas en las principales ciudades del país, (La Paz, Santa Cruz y Cochabamba) que conforman un eje económico, social y político con perspectivas positivas de desarrollo y vinculación con otras regiones y el exterior (BLANES, 2006).

Para los fines de este artículo nos centraremos en analizar los dos destinos fronterizos más importantes para la emigración boliviana: la Argentina y el Brasil. En el primer caso, sobre todo Buenos Aires, que como destino emigratorio boliviano es histórico; y en el segundo caso exclusivamente en Sao Paulo, será abordado con menos intensidad debido a la menor disponibilidad de información secundaria. Si bien en lo que va del presente siglo los estudios e investigaciones desarrollados en el país sobre la temática migratoria internacional son cada vez mayores y diversificados, hay que reconocer que aún existen significativos vacíos en diversos ámbitos de su tratamiento (impactos de las remesas, efectos de género y generacional, la variante étnica, lógicas de circulación, etc.), así como desarrollos desiguales en sus abordajes, siendo la presencia boliviana en la Argentina lo más trabajado.

La movilidad poblacional boliviana en busca de trabajo hacia Argentina tiene una historia de siglos. Tenemos antecedentes ya en el siglo XVIII donde muchas de las haciendas -desde Tucumán hacia el norte-, ocupaban mano de obra indígena y del "collado", como se denominaba a la población del actual territorio boliviano. En cierta medida, la economía del norte argentino estaba articulada a la economía miniera de Potosí (en el sur de Bolivia) en más de un sentido, situación que se mantuvo durante los primeros años de la república.

Hacia 1920 la migración de mano de obra boliviana hacia la Argentina comienza a aumentar en importancia. El fenómeno se da principalmente en el norte argentino, donde la industria azucarera -que antes se encontraba concentrada en la provincia de Tucumán- se expande hacia las provincias de Salta y Jujuy. En el censo argentino de 1947 casi el 88\% de los inmigrantes provenientes de Bolivia se establecieron en estas provincias, y sólo un 7\% se encontraba instalado en la provincia de Buenos Aires (GRIMSON, 1999). En décadas posteriores 
y debido al proceso de sustitución de importaciones, los movimientos poblacionales fueron concentrando su flujo hacia las áreas industriales que estaban situadas en las márgenes de las grandes ciudades, principalmente de Buenos Aires y dedicada al rubro de la construcción.

Ya en la década de los años ochenta el flujo de migrantes bolivianos aumentó, principalmente por la crisis económica boliviana que se vivió durante esos años y por la implementación del programa de ajuste estructural neoliberal llevado adelante desde 1985 en Bolivia. Estaba claro que los desplazamientos se habían reorientado hacia el centro urbano más importante en búsqueda de trabajo y mejores condiciones de vida. Los años noventa marcaron un periodo de estabilidad y en cierto modo de auge migratorio, en virtud de las características económicas de dolarización que asumió la república Argentina y también por la amnistía declarada en ese país (1999) que posibilitó la legalización de unos 110 mil bolivianos, la gran mayoría en Buenos Aires. Durante estos años se consolidaron y ramificaron muchas de las trayectorias migratorias anteriores y que correspondían al tipo urbano-urbano, tanto así, que fueron estas sólidas redes estructuradas las que amortiguaron los efectos de la crisis que vivió la Argentina hacia finales del 2001. En todo caso, el tiempo transcurrido tras la severa crisis argentina ha logrado estabilizar los flujos poblacionales entre estos dos países, aunque está claro que los niveles de ahorro y remesas característicos del periodo de dolarización se han diluido (BENENCIA, 2010 - 2011).

Alejandro Grimson (1999) detalla cómo los migrantes bolivianos asentados en Buenos Aires desarrollaron diversas estrategias, tanto para adquirir un trabajo, una vivienda y documentación, como para reunirse y construir en el nuevo contexto urbano lugares y prácticas de identificación; para él,

"[..] En Buenos Aires hay múltiples ámbitos de producción y re-construcción de identidades vinculados a la 'colectividad boliviana'. Es un tejido social diverso y disperso por distintas zonas de la ciudad que incluye bailantas, restaurantes, fiestas familiares y barriales, ligas de fútbol, programas de radio, asociaciones civiles, publicaciones, ferias y comercios de diferente tipo, dando cuenta de múltiples espacios vinculados con la bolivianidad [...]". (GRIMSON, 1999, p. 33-34)

Con el ánimo de subrayar algunos elementos mencionados por Grimson y que son fundamentales a la hora de generar interacciones con este contingente de migrantes debemos resaltar los procesos de 'territorialización transnacional' que se operan entre los dos países y cuyos horizontes culturales e identitarios definen un perfil propio que involucra a más de una generación. También es digno de destacar los niveles organizativos que despliegan los bolivianos en sus diversas actividades así como la incursión y permanencia en los medios de comunicación social, elementos que serán desarrollados más adelante.

Por su parte, el crecimiento geopolítico y económico del Brasil durante las últimas décadas ha convertido a este país en un polo de atracción de migrantes sudamericanos, condición que antes ostentaba casi en solitario la Argentina. La presencia boliviana en Sao Paulo no es un hecho nuevo; sin embargo, los estudios e investigaciones sobre esta presencia son muy escasos, tanto en el Brasil como en Bolivia. Podemos encontrar antecedentes ya en la década de los años 
cincuenta, pero será recién a finales de la década de los años ochenta cuando esta presencia se hace significativa, cobrando otro momento de fuerte crecimiento durante el primer decenio del presente siglo.

El perfil característico de los/as migrantes de Bolivia hacia el Brasil está dado por el hecho que en su mayoría son jóvenes de ambos sexos (si bien no se puede precisar porcentualmente la relación, es también evidente la sistemática incorporación de mujeres en dichas dinámicas), muchos de ellos solteros con escolaridad media y atraídos fundamentalmente por las promesas de buenos salarios en talleres textiles de empleadores que en los últimos años son bolivianos y que incursionaron en la industria de la confección textil, desplazando para ello a inmigrantes coreanos. Suelen ser oriundos de diversas partes de Bolivia pero sobre todo de zonas periurbanas de La Paz, El Alto y Cochabamba. La gran mayoría de estos inmigrantes se hallan en una situación migratoria de irregularidad (SILVA, 2006).

Es así que desde el punto de vista ocupacional, los y las bolivianos/as inmigrantes en el Brasil, desde los años ochenta, trabajan en su gran mayoría en el sector de la costura, por ser un segmento del mercado laboral que no exige experiencia previa ni edad mínima para la faena, lo cual le permite incorporar menores de edad sin mayor inconveniente y en condiciones de insalubridad ya que este ámbito de producción escapa a los controles y regulaciones del ramo.

Este modo de producción se enmarca en el modelo de 'acumulación flexible' del capital, donde la producción se da en base a la cantidad de prendas que el trabajador/a es capaz de costurar en el día. Resulta evidente también que las reglas de trabajo son permeadas por relaciones de parentesco y paisanaje que engloban y albergan relaciones de subordinación de la mano de obra, sobre todo la de mujeres. El producto final es vendido de manera general en los mercados locales de las ciudades, muchas de ellas bajo nombres de marcas muy conocidas (FREITAS, 2009).

En el caso de Brasil, uno de los autores que con mayor sistematicidad y dedicación ha investigado a los migrantes bolivianos es Sidney Antônio da Silva (1997, 2005) quien aborda desde una mirada antropológica la presencia poco conocida de la colectividad boliviana en Sao Paulo. Esto lo hace desde diversas entradas, privilegiando el tema de la inserción laboral en los talleres textiles de costura, donde el trabajo y la convivencia se dan en condiciones altamente precarias así como de indocumentación. Sin embargo, en la medida en que existe un fuerte involucramiento de la Iglesia Católica a través de las "Pastorales de Movilidad Humana" con la colectividad boliviana en este país, se cuenta también con cierta información proveniente de esta fuente. En los últimos dos años asistimos también al involucramiento de nuevos investigadores académicos que son atraídos sobre todo por la temática del trabajo textil que va en notable incremento (FREITAS, 2009).

Debemos destacar que este tipo de trabajo textil, en tanto nicho laboral transnacional que concentra mano de obra boliviana, está también presente en Buenos Aires. La historia y desarrollo de los talleres textiles allí como sector productivo tienen un fuerte impulso en los años ochenta, período definido como neoliberal donde variables como el desempleo, la reconversión económica, los procesos migratorios en busca de trabajo y mejores oportunidades de vida, como producto directo de las crisis y estallidos sociales en la región, alcanzan su momento de constitución en la década de la década de los noventa. 
Respecto a los talleres, los talleristas y los trabajadores, Jorge Vargas (2010) nos advierte para el caso de Buenos Aires que responde al mismo patrón que Sao Paulo, sobre lecturas y aproximaciones controversiales que se generan cuando se habla del trabajo en los talleres de costura, a partir de nociones como "trabajo esclavo", "en negro", "marginal" e "informal". Dichas interpretaciones tienden a soslayar la complejidad, los cambios e hibridaciones de los procesos en los cuales intervienen y construyen de modo cotidiano los sectores migrantes involucrados, en procesos colectivos que por otra parte ya suman más de dos décadas de desarrollo en la actividad productiva laboral y económica local. Utilizar el término esclavo para referirse a una actividad productiva inserta en un sistema social y en un modo de producción del capital no permite generar herramientas de conocimiento adecuadas y productivas. Algo de ello ocurrió con el abordaje de situaciones presentes en los talleres textiles. De ahí que se debe partir desde otros puntos de vista, con otras herramientas conceptuales, abordar la situación como procesos complejos y cambiantes, evitando reducirlas al campo de la simplificación y el prejuicio.

\section{EL TRABAJO BOLIVIANO EN LOS TALLERES TEXTILES DE BUENOS AIRES Y SAN PABLO}

La historia y desarrollo de los talleres textiles en la Argentina como sector productivo con las características actuales comienza en los años ochenta, período definido como neoliberal en términos económico-políticos, donde variables como el desempleo, la reconversión económica, los procesos migratorios en busca de trabajo y mejores oportunidades de vida, como producto directo de las crisis y estallidos sociales en la región, y alcanzan su momento de constitución en la década de los noventa, decenio gobernado por Menem, gobierno que a nivel local realizó la aplicación de las políticas impulsadas a partir de las premisas diseñadas por el Consenso de Washington: retiro activo y reducción del Estado, cierre masivo de fuentes de empleo, reconversión económica hacia la especulación financiera. etc.

Durante el 2010 la economía volvió a expandirse significativamente, registrando un crecimiento interanual del 9,2\%, bastante por encima del promedio de la región. Dicho crecimiento trajo aparejada una disminución de la tasa de desocupación, que pasó del 8,4\% en el último trimestre de 2009 al 7,3\% en el mismo período de 2010 . En el primer trimestre de 2011 el crecimiento interanual del PIB fue del 9,9\%, lo que implica un piso de crecimiento del 6,1\% para el resto del año. La mayor inmigración regional procede desde Bolivia.

En el año 2006 un incendio que acabó con la vida de seis personas sacó a la luz las abusivas condiciones de trabajo en una red de plantas textiles clandestinas de Buenos Aires. Las dos mujeres y cuatro niños que perdieron la vida estaban encerrados dentro del taller textil. La Unión de Trabajadores Costureros (UTC), una asamblea de trabajadores indocumentados del sector textil, ha denunciado en los últimos años más de 8.000 casos de abusos laborales dentro de los casi 400 talleres textiles clandestinos de la ciudad. Unos 100.000 inmigrantes indocumentados trabajan en esas plantas de producción inseguras, con un salario medio, cuando cobran, de 100 dólares mensuales.

Respecto a los talleres, los talleristas y los trabajadores Jorge Vargas (2010) nos advierte sobre lecturas y aproximaciones muy controversiales que se generan cuando se habla del trabajo 
en los talleres de costura, a partir de nociones como las de: "trabajo esclavo", "en negro", "marginal e informal", dichas interpretaciones tienden a soslayar la complejidad, los cambios e hibridaciones de los procesos en los cuales intervienen y construyen de modo cotidiano los sectores migrantes involucrados, en procesos colectivos que por otra parte ya suman mas de dos décadas de desarrollo en la actividad productiva, laboral y económica local. Utilizar el término esclavo para referirse a una actividad productiva inserta en un sistema social y en un modo de producción del capital no permite generar herramientas de conocimiento adecuadas y productivas. Algo de ello ocurrió con el abordaje de situaciones presentes en los talleres textiles. Ante una tragedia como el incendio del taller de Caballito, la mirada generalizada posterior fue encontrar el mismo tipo de situación de explotación laboral y humana en la actividad productiva en general. Desde entonces todo taller textil en el que participan ciudadanos de origen boliviano es sospechado, si no culpable, de ser esclavista.

Insistimos que no se trata de desconocer u ocultar la existencia de ese tipo de abusos, de lo que se trata es de abordarlos desde el conocimiento por un lado, para comprender los procesos que conducen a esa condición y para quienes deban generar acciones institucionales desde el ámbito público, lo hagan generando soluciones efectivas y reales como ciudadanos, trabajadores, pequeños empresarios y atendiendo al sentido de dignidad y autonomía.

El otro punto señalado por Vargas es la "criminalización de la actividad productiva". Esto ocurre con la actividad de los talleres textiles, en términos generales se criminalizó la actividad laboral del sector; ante la actividad delictiva de una parte, esa modalidad se trasladó a todo el sector como condición asociada.

De ahí que se deben partir desde otros puntos de vista, con otras herramientas conceptuales, abordar la situación como procesos complejos y cambiantes, evitando reducirlas al campo de la simplificación y el prejuicio.

Una de las causas de que muchas unidades productivas textiles deban estar obligadas a apelar a grados de informalidad laboral o tributaria es porque en la distribución de ganancias y beneficios de todo el sector, estos favorecen abiertamente a los fabricantes y grandes marcas y no al sector laboral. La ausencia del Estado para equilibrar esta desigualdad e injusticia es notoria. No se trata de que no se hayan establecido precios y parámetros en las negociaciones colectivas. Se trata de que estas aún no lleguen al sector de talleristas y trabajadores, y que en la realidad están sujetos al interés y arbitrio de intermediarios y fabricantes.

Magliano (2007) señala que la migración de mujeres bolivianas hacia Argentina ha sido históricamente parte de un proyecto migratorio familiar, y si bien en los últimos años aumentó el número de mujeres que arribaron solas, todavía la mayor parte de este flujo se desplaza hacia este país en contextos familiares, de ahí la importancia de la familia para analizar el rol de la mujer boliviana en este proceso, y la persistencia de relaciones de género desiguales en ámbitos familiares y sociales.

Asimismo, cuando la mujer se mueve como integrante de un grupo familiar, cuando la familia ejerce control a través de las redes sociales o parentales, cuando no disponen de sus ingresos, o cuando son indocumentadas y ocupan puestos de trabajo en el sector informal de la economía, son mayores las probabilidades de que no se modifiquen los roles de género (HUGO, 
2000). Además, las actividades productivas y los aportes económicos que realizan las migrantes bolivianas no generan necesariamente una autonomía e independencia en el ámbito de la familia, esto va a depender, en muchas ocasiones, del tipo de trabajo que llevan a cabo estas mujeres y de la forma en que lo obtienen. En efecto, la estratificación de género, etnia y clase en los mercados de trabajo en las comunidades de arribo profundiza la desigualdad, discriminación y exclusión que afecta a esta población, situación que refuerza también los roles de género.

Por su parte Tanja Bastia (2010) en su texto sobre las relaciones de género y los cambios sociales en la migraciones urbanas para el caso de Cochabamba señala que "[...] la inserción laboral de los emigrantes bolivianos en Buenos Aires mostró que fue más fácil para las mujeres conseguir trabajo en el periodo inmediato a su llegada [...]” (BASTIA, 2010, p. 77). Establece también que en el caso de las manufacturas textiles de Buenos Aires, si bien se emplean en igual porcentaje hombres y mujeres, pareciera que en los últimos años habría un leve aumento de las mujeres.

Del total de encuestados, el $45 \%$ trabajaron en talleres textiles, siendo este oficio una opción mucho más importante para las mujeres que para los hombres, ya que más de la mitad de ellas $(52,5 \%)$ se habían sustentado principalmente por medios de este oficio mientras sólo lo habían hecho así aproximadamente un tercio de los varones encuestados. Según la encuesta, en este sector los hombres ganaban el doble del sueldo mensual de las mujeres. (BASTIA, 2010, p. $80)$.

Si bien los procesos migratorios permiten a las mujeres bolivianas opciones de generación de recursos económicos de manera autónoma reposicionándolas desde un rol más activo en el seno de sus familias, también parece ser que su inserción laboral tiende a ser provisional lo cual incide en cierta medida en sus retornos a la comunidad.

Por su lado la economía brasileña demostró en 2010 su vigor, al recuperarse rápidamente del impacto de la crisis internacional, con un crecimiento de su producto interno bruto (PIB) del $7,5 \%$, el mayor observado desde 1986. Esa expansión se produjo gracias a la respuesta del mercado interno y a la recuperación de las exportaciones. Sin embargo, el aumento de la demanda interna y el alza de los productos básicos en el comercio exterior influyeron en la inflación, que en 2010 alcanzó el 5,9\%, cifra que las autoridades económicas consideraron una amenaza, dada su cercanía al límite superior de la meta inflacionaria, fijado en el 6,5\%.

El crecimiento geopolítico y económico brasileño convierte a este país en un polo de atracción de migrantes sudamericanos, que antes ostentaba Argentina. A medida que la economía de Brasil siga creciendo, es probable que se abran nuevas fábricas para sustituir a las que cierren las autoridades. Así, todavía parece fácil atraer a los inmigrantes pobres a las grandes ciudades con promesas de una vida más digna y un futuro mejor.

La presencia boliviana en San Pablo no es un hecho nuevo. Podemos encontrar antecedentes ya en la década de los años cincuenta, pero será recién a finales de la década de los años ochenta cuando esta presencia se hace significativa cobrando otro momento de crecimiento en los últimos años. El perfil característico de los/as imigrantes de Bolivia está dado por el hecho que en su mayoría son jóvenes de ambos sexos (si bien no se puede precisar porcentualmente la relación es también evidente la sistemática incorporación de mujeres en dichas dinámicas), 
muchos de ellos solteros con escolaridad media y atraídos fundamentalmente por las promesas de buenos salarios en talleres textiles de empleadores coreanos, pero que en los últimos años muchos bolivianos también incursionaron en la industria de la confección. Suelen ser oriundos de diversas partes de Bolivia pero sobre todo de zonas periurbanas de La Paz, El Alto y Cochabamba. La gran mayoría de estos inmigrantes se hallan en una situación migratoria de irregularidad.

Desde un punto de vista ocupacional, los y las bolivianos/as inmigrantes en el Brasil, desde los años ochenta, trabajan en su gran mayoría en el sector de la costura, por ser un segmento del mercado laboral que no exige experiencia previa ni edad mínima para la faena, lo cual le permite incorporar menores de edad sin mayor inconveniente y en condiciones de insalubridad ya que esta ámbito de producción escapa a los controles y regulaciones del ramo. Este modo de producción se enmarca en el modelo de 'acumulación flexible' del capital, donde la producción se da en base a la cantidad de prendas que el trabajador/a es capaz de costurar en el día. Resulta evidente también que las reglas de trabajo son permeadas por relaciones de parentesco y paisanaje que engloban y albergan relaciones de subordinación de la mano de obra, sobre todo el de mujeres. El producto final es vendido de manera general en los mercados locales de las ciudades, muchas de ellas bajo nombres de marcas muy conocidas.

\section{BIBLIOGRAFIA}

BENENCIA, Roberto. Los inmigrantes bolivianos, ¿sujetos de agenda política en la Argentina? In: FELDMAN-BIANCO, Bela; RIVERA SÁNCHEZ, Liliana; STEFONI; Carolina; VILLA MARTÍNEZ, Marta Inés (Compiladoras). La construcción social del sujeto migrante en América latina: prácticas, representaciones y categorías. Ecuador: CLACSO-FLACSO-Ediciones Universidad Alberto Hurtado, 2011.

BENENCIA, Roberto. El infierno del trabajo esclavo: la contratara de las 'exitosas' economías étnicas. In: GARCÍA, Antonio Agustín; GADEA, María Elena; PEDREÑO, Andrés (Eds.), Tránsitos migratorios: contextos transnacionales y proyectos familiares en las migraciones actuales. Murcia: Ediciones de la Universidad de Murcia, 2010.

BLANES, José. Bolivia. Áreas metropolitanas en clave de desarrollo y autonomía. La Paz: ILDIS, 2006.

CONDARCO, Ramiro. El escenario andino y el hombre. La Paz: Renovación, 1971.

DIRECIÓN GENERAL DE RÉGIMEN CONSULAR (DGRC). Informe Anual, 2010 (mimeo).

FREITAS, Patrícia Tavares. Imigração e Experiência Social: o circuito de subcontratação transnacional de força-de-trabalho boliviana para o abastecimento de oficinas de costura na cidade de São Paulo. Tesis de Maestría, Instituto de Filosofía y Humanidades, Universidad Estadual de Campinas, 2009. 
GRIMSON, Alejandro. Relatos de la diferencia y la igualdad. Los bolivianos en Buenos Aires. Buenos Aires: Eudeba, 1999.

INSTITUTO NACIONAL DE ESTADISTICA (INE). Censo Nacional de Población y Vivienda, 2014.

MURRA, John. Formaciones económicas y políticas en el mundo andino. Lima: IEP, 1975.

ORGANIZACIÓN INTERNACIONAL PARA LAS MIGRACIONES (OIM). Perfil Migratorio de Bolivia. Buenos Aires: OIM Oficina Regional para América del Sur, 2011.

ORGANIZACIÓN INTERNACIONAL PARA LAS MIGRACIONES (OIM). Relevamiento y diagnóstico de las asociaciones de la colectividad boliviana en Argentina. Informe final. Buenos Aires: OIM-CEMLA, 2004.

SILVA, Sidney. Bolivianos em São Paulo: entre o sonho e a realidade. In: Estudos Avançados, v. 20, n. 57, pp. 157-170, 2006.

SILVA, Sidney. Inmigrantes no Brasil. Bolivianos, a presença da cultura andina. Sao Paulo: Compañía Editorial Nacional, 2005.

VARGAS, Jorge. Talleristas y trabajadores. Mi razón no pide piedad, 2010 (mimeo). 